\title{
Neuralgic Amyotrophy: a Clinical and Electromyographic Study
}

\author{
J. L. JAMES,* M.D., B.SC., M.R.C.P. ; D. W. MILES, † M.B., B.sC., M.R.C.P.
}

Brit. med. F., 1966, 2, 1042-1043

The clinical syndrome of neuralgic amyotrophy is strikingly stereotyped. It consists of severe burning pain of sudden onset in the outer aspect of the arm accompanied by wasting and weakness of the muscles of the upper limb. Affected muscles may be completely paralysed. The pain is occasionally transient, but usually lasts up to six weeks, and sometimes even longer. Weakness or paralysis follows its onset in the space of one to four days in $80 \%$ of cases. The motor symptoms are usually maximal at the outset, but progression over a period of seven days may be observed. The site of the pain and the muscles affected are not necessarily correlated. Paralysed muscles are flaccid and waste rapidly, but fasciculation is not obtrusive. Sensory changes occur most commonly when the circumflex nerve is affected. In the majority of patients systemic disturbances-for example, malaise, fever-are minimal (Turner, 1944 ; Parsonage and Turner, 1948).

Muscles affected are usually serratus anterior (Richardson, 1942), spinati, deltoid, and trapezius (Spillane, 1943), but any muscle of the arm may be deranged and bilateral involvement is quite common. Wyburn-Mason (1941) encountered 42 cases in a period of nine months, chiefly during the summer. Spillane (1943) described 46 cases in Service men, 26 of whom developed the disorder while they were in hospital for some other reason. Other authors have noticed a close association with hospital (Parsonage and Turner, 1948), and French workers have often considered injections of serum-with brisk serum reactions-to be responsible (Pollet, 1924). This is not by any means an invariable experience (Richardson, 1942). Both sexes are affected; in the earlier papers males preponderate in the ratio of $12: 1$, but Turner and Parsonage (1957) included nearly as many women as men. Age ranges of 30-79 (WyburnMason, 1941) and 16-54 years (Turner, 1944) have been quoted.

Prognosis has been said to be poor where there is much muscle-wasting (Spillane, 1943 ; Turner, 1944), but Dixon and Dick (1945) described recovery taking up to two years in 13 out of 16 patients who had been severely affected. Turner and Parsonage (1957) agreed that return of power was the rule, the time needed for recovery varying with the nerves affected. Recurrent attacks have been described (Dixon and Dick, 1945).

The site of the lesion responsible for the disorder has been variously suggested as the anterior primary rami of lower cervical and upper thoracic roots (Wyburn-Mason, 1941), with trunks of peripheral nerves affected in addition to or in isolation from the roots (Turner, 1944) and sometimes the spinal cord itself (Parsonage and Turner, 1948). No specific evidence for these conclusions has been offered. Necropsy evidence is lacking.

The cause of the neuronal damage has been thought to be an inflammatory reaction of infectious or allergic origin.

Electromyography may give some information regarding the site if not the aetiology of the lesion, and a group of patients with neuralgic amyotrophy have been examined in this way.

\section{Material and Methods}

Between April 1961 and March 196438 patients were referred to the Department of Electromyography, the General Infirmary at Leeds. A clinical history was obtained from each patient - Tutor in Medicine, Unlversity of Leeds. Now Consultant Physician
to the Huddersfield Hospitals.

t Lecturer in Medicine, University of Leeds. and clinical examination was carried out. Electromyograms were obtained from affected muscles by means of a concentric coaxial needle electrode. Spontaneous potentials in resting muscles and the action potentials produced on attempted full voluntary contraction were displayed on the screen of a Tektronix 502 oscilloscope. Special attention was paid to the presence of fibrillation potentials or "positive waves" when muscles were at rest, and to the interference pattern of the action potentials recorded from contracting muscles for information on the question of the number of motor units at work, their size, and the characteristics of their innervation.

Assessment of the severity of the disability produced by the neuralgic amyotrophy was made in two ways: (a) from the period during which the patient was unable to carry out his or her normal work, and $(b)$ from the number of muscles affected and the extent of the weakness of each, using the criteria of the M.R.C. (1943). If the power was graded 0-3 in any muscle the disability was described as severe; if grade 4 in more than two muscles, as moderate; if grade four in one or two muscles, as mild; and as nil if there was no objective clinical evidence of weakness.

Twenty-nine patients presented themselves for review after an interval, and clinical examination was repeated; electromyography was repeated in $28 . X$-ray examination of the cervical spine was carried out in 23 patients.

\section{Results}

There were 30 men and eight women in our group of patients. Their ages ranged from 9 to 75 years, and, while there were more patients in the fifth and sixth decades than in the others, the distribution appeared to be random. Onset of symptoms had occurred in all months of the year except June.

Pain was bilateral in eight patients, on the left only in 16, and on the right only in 14 . It was experienced most commonly in the shoulders and around the scapula-particularly its medial border-but the whole arm was affected in three patients, the forearm only in one, and the hand only in another. Twenty-three patients had pain severe enough to disturb sleep for up to four weeks ; in a further 13 it lasted up to 12 weeks and in two for up to six months. However, the mean for the whole group was five weeks.

Muscle-weakness involved the deltoid most often (33 cases) and the supraspinatus and infraspinatus very commonly (23 examples of each). All the muscles of the upper limb except the long finger flexors were affected on occasion.

Sixteen patients exhibited sensory involvement. It affected the circumflex-nerve distribution in 12 , the C 5-6 dermatomes in two, and had a glove distribution in two.

Nine patients, all male, had had recent contact with hospitals, dentists, or doctors, but only two had had serum injections, and neither of these experienced any untoward reaction.

\section{Findings on Electromyography}

Only one patient out of 36 had a normal electromyogram when first examined. Fibrillation potentials-brief spontaneous low-voltage discharges due to the contraction of individual muscle fibres as a result of loss of their nerve supply-were 
recorded from 23 patients; three showed a reduction in the number of active motor units in the muscles; and nine showed a reduction in the size of the motor units. All of these changes implied disturbance of the nerve supply of the muscles affected.

A satisfactory follow-up examination was achieved in 29 patients. The initial disability was mild in 12 , moderate in 10 , and severe in 7 . After periods ranging between 4 and 35 months 18 patients had no disability, but eight remained mildly and three moderately disabled. All patients showed improvement, and this was most rapid and complete in those who had the least initial disturbance. Of 10 patients who were under observation for more than two years two had persistent disability. There was poor correlation between the time that each patient spent off work and the apparent clinical severity of his illness. Twenty-three patients developed muscle-wasting, but this did not preclude a good prognosis. The electromyogram returned to normal in 17 out of 28 patients examined and none showed absence of electrical activity on voluntary muscular contraction, though eight had been unable to contract their affected muscles at all at the initial examination.

Total recovery or a very substantial degree of improvement was seen in over $90 \%$ of patients. In some it was achieved in less than a year, but in the majority it took between one and two years.

\section{Discussion}

\section{Site of the Anatomical Lesion}

When muscle fibres lose their nerve supply they become unduly sensitive to circulating acetylcholine and contract spontaneously (Denny-Brown and Pennybacker, 1938). The resulting electrical activity which can be recorded from resting muscles is described as fibrillation. It is always pathological. Damage to nerves also alters the pattern of voluntary electrical activity in muscles (Taverner, 1961). Muscle contraction depends upon the asynchronous excitation of a large number of motor units, and each motor unit produces an electrical action potential. (A motor unit consists of a nerve cell and all the muscle fibres it innervates.) Where whole motor units are lost, the electrical interference pattern of action potentials of a contracting muscle becomes less complex until the contraction of individual units may be seen. In a chronic denervation process the fibres of surviving nerve cells may sprout to innervate muscle fibres which have become isolated from the nervous system, producing "giant" motor units with correspondingly large action potentials when the muscle fibres contract. In general this is more common in diseases affecting anterior horn cells in the spinal cord than in those affecting peripheral nerves. Under different circumstances the number of motor units may not be substantially depleted, but their action potentials diminish in size because fewer muscle fibres are included in the unit. This may happen in disease processes, such as polyneuritis, where the peripheral parts of nerve fibres are chiefly affected.

The pattern of electrical activity recorded by electromyography therefore has some localizing value. The combination of this with clinical findings enabled us to locate the lesions in 26 patients as follows: nerve roots, 11 ; peripheral parts of nerve fibres, 6 ; single peripheral nerve, 4 ; multiple peripheral nerves, 5. We did not find any evidence of damage within the spinal cord.

\section{Prognosis}

It appears that neuralgic amyotrophy is due to damage to axons at various distances from their cells of origin and that those arising from the fifth and sixth cervical segments are particularly vulnerable. However, the prognosis is uniformly good no matter where the damage occurs, but recovery will take longer with more proximally situated lesions. Occasionally the regeneration of nerve fibres from their proximal ends takes place in the wrong direction. We have seen one patient with a C 5 lesion which must have affected both his deltoid and his diaphragm. In the process of regeneration some of the fibres of his phrenic nerve grew into the neurilemmal tubes of his circumflex nerve, with the result that his deltoid contracted every time he breathed in. Despite this his functional recovery was satisfactory.

\section{Aetiology of the Lesion}

We have been unable to involve sex, serum injections, contact with hospitals, military service, or season of the year in aetiology. The age range of our patients, as of others, is very wide. One of the commonest referral diagnoses in our patients was cervical spondylosis. Twenty-three of our patients had $x$-ray films taken of the cervical spine. One of these showed marked spondylosis, and neurosurgical treatment of this condition had been contemplated; three more showed small osteophytes, but $19 x$-ray films were normal.

There is thus no definite clue to the cause of this condition. However, we have been struck by certain resemblances between neuralgic amyotrophy and Bell's palsy, as described by Taverner in recent years (Taverner, 1955, 1959 ; Langworth and Taverner, 1963): the sudden onset with pain, the rapid recovery in some where denervation is not severe, and the more prolonged recovery in others, leaving a few with a measure of persistent disability and "associated movements." Such a resemblance does not clarify the problem of aetiology, but even in such a condition as Bell's palsy therapeutic possibilities exist and the mitigation of neuronal damage by early administration of A.C.T.H. has been reported (Taverner et al., 1966). It is possible that similar measures would be profitable in patients with neuralgic amyotrophy.

\section{Summary}

The clinical and electromyographic features of 38 patients who developed neuralgic amyotrophy are reported. Twentynine of these were reviewed after an interval of from six months to two years, depending on the severity of the attack.

The lesion was sometimes located in the proximal and sometimes in the distal part of the neuron, and on occasion in one or more peripheral nerves.

The cause of the condition is unknown and no relation with injections, contact with hospitals, or military service was observed in this group. Radiography of the cervical spine was normal in 19 of the 23 patients in whom it was performed.

The prognosis was good in $93 \%$ of the patients.

We are grateful to the many physicians and surgeons who referred patients to the E.M.G. Clinic. We are indebted to Dr. D. Taverner for his help and advice during this study.

\section{REFERENCES}

Denny-Brown, D., and Pennybacker, J. B. (1938). Brain, 61, 311.

Dixon, G. J., and Dick, T. B. S. (1945). Lancet, 2, 707.

Langworth, E. P., and Taverner, D. (1963). Brain, 86, 465.

Medical Research Council (1943). W ar Memo. No. 7, p. 1.

Parsonage, M. J., and Turner, J. W. A. (1948). Lancet, 1, 973.

Pollet, L. (1924). Gaz. Hôp. (Paris), 97, 561.

Richardson, J. S. (1942). Lancet, 1, 618.

Spillane, J. D. (1943). Ibid., 2, 532.

(1959). Proc. roy. Soc. Med., 52, 1077.

(1961). In Electrodiagnosis and Electromyography, edited by $\mathrm{S}$ Licht, 2nd ed., chap. 13. Licht, New Haven, Conn.

Fearnley, M. E., Kemble, F., Miles, D. W., and Peiris, O. A. (1966). Brit. med. Y., 1, 391

Turner, J. W. A. (1944)., Ibid., 2, 592.

and Parsonage, M. J. (1957). Lancet, 2, 209.

Wyburn-Mason, R. (1941). Ibld., 2, 662 . 